\title{
Effects of Berberine on the Gastrointestinal Microbiota
}

\author{
Lichao Zhang ${ }^{1,2,3+}$, Xiaoying $W u^{1,2,4 \dagger}$, Ruibing Yang ${ }^{5}$, Fang Chen ${ }^{6}$, Yao Liao ${ }^{1,2,3}$, \\ Zifeng Zhu ${ }^{1,2,3}$, Zhongdao Wu ${ }^{1,2,3}, X^{\text {i Sun }}{ }^{1,2,3^{*}}$ and Lifu Wang ${ }^{1,2,3^{*}}$ \\ ${ }^{1}$ Department of Parasitology of Zhongshan School of Medicine, Sun Yat-sen University, Guangzhou, China, 2 Key Laboratory \\ of Tropical Disease Control, Ministry of Education, Sun Yat-sen University, Guangzhou, China, ${ }^{3}$ Provincial Engineering \\ Technology Research Center for Biological Vector Control, Guangzhou, China, ${ }^{4}$ Department of Gastroenterology, Third \\ Affiliated Hospital of Sun Yat-sen University, Guangzhou, China, ${ }^{5}$ Medical Department, Xizang Minzu University, Xianyang, \\ China, ${ }^{6}$ School of Medicine, South China University of Technology, Guangzhou, China
}

\section{OPEN ACCESS}

Edited by:

Jaime Garcia-Mena,

Instituto Politécnico Nacional de México (CINVESTAV), Mexico

Reviewed by: Bruno Melo Carvalho, Universidade de Pernambuco, Brazil Guilherme Zweig Rocha, Campinas State University, Brazil

${ }^{*}$ Correspondence: Xi Sun

sunxi2@mail.sysu.edu.cn Lifu Wang wanglf29@mail.sysu.edu.cn

${ }^{\dagger}$ These authors have contributed equally to this work

Specialty section:

This article was submitted to Microbiome in Health and Disease, a section of the journal

Frontiers in Cellular and Infection Microbiology

Received: 21 August 2020 Accepted: 31 December 2020 Published: 19 February 2021

Citation:

Zhang L, Wu X, Yang R, Chen F, Liao $Y$, Zhu Z, Wu Z, Sun $X$ and Wang $L$ (2021) Effects of Berberine on the Gastrointestinal Microbiota. Front. Cell. Infect. Microbiol. 10:588517. doi: 10.3389/fcimb.2020.588517
The gastrointestinal microbiota is a multi-faceted system that is unraveling novel contributors to the development and progression of several diseases. Berberine has been used to treat obesity, diabetes mellitus, atherosclerosis, and metabolic diseases in China. There are also clinical trials regarding berberine use in cardiovascular, gastrointestinal, and endocrine diseases. Berberine elicits clinical benefits at standard doses and has low toxicity. The mechanism underlying the role of berberine in lipidlowering and insulin resistance is incompletely understood, but one of the possible mechanisms is related to its effect on the gastrointestinal microbiota. An extensive search in electronic databases (PubMed, Scopus, Embase, Web of Sciences, Science Direct) was used to identify the role of the gastrointestinal microbiota in the berberine treatment. The aim of this review was to summarize the pharmacologic effects of berberine on animals and humans by regulation of the gastrointestinal microbiota.

Keywords: berberine, intestinal flora, obesity, diabetes, hyperlipidemia

\section{INTRODUCTION}

Berberine is a quaternary ammonium salt from the protoberberine group of isoquinoline alkaloids (Caliceti et al., 2016). The latter are present in the roots, bark, and other structures of plants found typically in traditional Chinese/East Asian medicines. Such plants include Coptis chinensis, Berberis aristata, B. petiolaris, B. vulgaris, B. aquifolium, and B. thunbergii (Cicero and Baggioni, 2016). Unusually high berberine content has been reported in Phellodendron amurense and C. chinensis (Habtemariam, 2016).

Many people in China use traditional herbal formulations to treat diseases, as recorded in the Pharmacopoeia of China (2015). Such formulations have excellent efficacy, including clearing away heat, resolving dampness, purging fire, and detoxification (Wang et al., 2017a). These formulations have been used to treat pharyngolaryngitis, typhoid, gastroenteritis, diabetes mellitus (DM), and secretory diarrhea for more than 1,000 years in China (Yang et al., 2010).

Berberine and its derivatives display several pharmacologic effects through various mechanisms (Jin et al., 2016). Berberine may be therapeutic against various types of chronic diseases, such as obesity, DM, inflammatory bowel disease (IBD), atherosclerosis, Alzheimer's disease, rheumatoid arthritis, and cardiovascular diseases, due to its multiple-target effects (Jin et al., 2016). Also, the binding of berberine 
with histone-DNA complexes can cause interferences in vital cellular processes, such as cell division and cause the death of cancer cells by activating the apoptosis in living cells (Chen et al., 2016; Hasanein et al., 2017; Roudini et al., 2019). In vitro, berberine has important anti-inflammatory and antioxidant activities.

In animal models, berberine has neuro-protective and cardiovascular-protective effects. Tan et al. showed that berberine was distributed rapidly (in a descending order of its amount) in the brain, lungs, heart, liver, kidneys, muscle, pancreas, and fat in rats (Tan et al., 2013). The mechanism of action of berberine is associated with its regulatory effect on cellular targets, such as the low-density lipoprotein receptor (LDLR), insulin receptor (IR), adenosine monophosphateactivated protein kinase(AMPK), proprotein convertase subtilisin kexin-9, protein tyrosine phosphatase-1B(PTP-1B), mitochondrial adenosine triphosphate (ATP) production, and brown fat tissue (Kong et al., 2004).

\section{THE GASTROINTESTINAL MICROBIOTA (GM)}

The GM (also referred to as the "gut flora" or "gut microbiota") are the microorganisms that live in the digestive tracts of mammals, including bacteria, archaea, viruses, fungi, and some parasites. Most of the GM reside in the distal large intestine. Dysbiosis refers to an alteration in the quality and/or quantity of the GM; such changes can influence the physiology of the host and lead to the onset of various diseases (Kuno et al., 2016).

Research carried out over the last few years indicates that the GM represents an important factor for the regulation of body health and may be closely related to the pathogenesis of obesity (Walker and Parkhill, 2013), diabetes mellitus (DM), inflammation (Kamada et al., 2013), cardiovascular diseases and cancer (Louis et al., 2014), and other diseases (Round and Mazmanian, 2009). The GM is composed of several phyla, including Bacteroidetes, Firmicutes, Proteobacteria, Verrucomicrobia, Actinobacteria, Fusobacteria, and Cyanobacteria et al (Sekirov et al., 2010). Bacteroidetes and Firmicutes are known to represent the main components of the GM. An imbalance in the ratio of Firmicutes and Bacteroidetes (F:B) in the GM has been associated with several diseases (Gao et al., 2017). Recent evidence also suggests that the GM plays a role in homeostasis and may exert positive influence on immune responses and prevent the development of inflammatory diseases. Berberine has been shown to affect the bacteria that produce short-chain fatty acids (SCFAs) in the GM (Wang et al., 2017b). Research has also shown that SCFA-producing bacteria benefit the host by protecting the mucosa from damage induced by pathogens, by providing colonocyte nutrients, and by mitigating inflammation (Maslowski et al., 2009).

\section{THE EFFECTS OF BERBERINE ON THE GM}

The GM is also known to affect drug metabolism, both directly and indirectly, and particularly with regards to drugs that are administered orally. Berberine reduces the levels of lipids and glucose in the blood via multi-target mechanisms, including modulation of the GM composition (Zhang et al., 2012). Berberine is also known to reduce the diversity of the GM and interfere with the relative abundance of Desulfovibrio, Eubacterium, and Bacteroides (Cui et al., 2018b). In addition, Bacteroides were shown to be enriched in the colon and terminal ileum of mice $(\mathrm{C} 57 \mathrm{BL} / 6)$ treated with berberine, but berberine treatment reduced the populations of Ruminococcus gnavu (Genus of Mediterraneibacter), Ruminococcus schinkii (Genus of Blautia), Lactobacillus acidophilus (Genus of Lactobacillus), Lactobacillus murinus (Genus of Ligilactobacillus), and Lactococcus lactis (Genus of Lactococcus) (Guo et al., 2016). Recent studies have shown that berberine has beneficial effects on the immune cells of the intestinal immune system and affects the expression of several intestinal immune factors. Berberine has also been shown to inhibit the mRNA expression of interleukin (IL)-1 $\beta, I L-4, I L-10$, macrophage migration inhibitory factor $(M I F)$, and tumor necrosis factor (TNF)- $\alpha$, while also reducing low-grade inflammation (Gong et al., 2017). Short-term exposure to berberine alters the populations of intestinal bacteria by reducing the activity of Clostridium cluster XIVa and IV, and their bile salt hydrolase (BSH), thus leading to the accumulation of taurocholic acid (TCA). TCA can activate intestinal farnesoid $\mathrm{X}$ receptor (FXR) which can then mediate the metabolism of bile acids, lipids, and glucose (Tian et al., 2019). Butyrate is a short-chain fatty acid (SCFA) produced during fermentation of fibers and other substrates by an anaerobic bacteria resident in the gastrointestinal tract (Roediger et al., 1982). Berberine has also been shown to enrich the population of butyrate-producing bacteria in the GM, thus promoting the synthesis of butyrate via the acetyl CoA-butyryl CoA-butyrate pathway. Subsequently, the butyrate enters the blood and reduces the levels of lipids and glucose (Wang et al., 2017b).

The GM is known to play a key role in the development of metabolic disorders. One factor underlying the application of berberine treatment is that berberine can increase the rates of cellular glucose uptake and metabolism (Cok et al., 2011). Other research studies are investigating the effects of berberine against cancer. In this article, we review the role of the GM on nontransmissible diseases following berberine treatment.

\section{THE EFFECTS OF BERBERINE ON OBESITY}

The global obesity epidemic is prompting significant efforts to identify host and environmental factors that affect energy balance in the human body. For example, Turnbaugh et al. reported that host obesity is related to an increase in the intestinal F:B ratio (Turnbaugh et al., 2006). Berberine has been shown to revert the structural changes in the GM induced by a high-fat diet and regulate diversity in the GM. Berberine has also been shown to change 134 operational taxonomic units (OTUs) that were identified by nearest 
shrunken centroids analysis in obese rats induced by a high-fat diet, and was also associated with changes in obesity phenotypes. Sixty of the 134 OTUs were significantly increased with berberine treatment, particularly those belonging to putative SCFA-producing bacteria, including Allobaculum, Bacteroides, Blautia, Butyricicoccus, and Phascolarctobacterium.

A previous study showed that there is a reduced abundance of A. muciniphila in obese humans (Everard et al., 2013). Interestingly, others discovered that A. muciniphila was less abundant in the intestinal microbiota of both genetic and dietinduced obese and diabetic mice (Schneeberger et al., 2015; LealDiaz et al., 2016). Akkermansia spp. abundance was markedly increased in HFD-fed mice treated with berberine (Zhu et al., 2018). A. muciniphila treatment was shown to reverse metabolic disorders induced by high-fat diets, including fat-mass gain, metabolic endotoxemia, adipose tissue inflammation, and insulin resistance (IR). The administration of A. muciniphila was also shown to increase the intestinal levels of endocannabinoids that control inflammation, the gut barrier, and the secretion of peptides in the gut (Everard et al., 2013). Increased levels of colonization by $A$. muciniphila were also shown to induce the expression of lowdensity lipoprotein receptors and apolipoprotein $\mathrm{E}$ in the hepatocytes of CREBH-null mice (C57BL/6J). This facilitated the uptake of intermediate-density lipoprotein via the mediation of apolipoprotein B100 and apolipoprotein E, thus leading to the increased clearance of triglyceride-rich lipoprotein remnants, chylomicron remnants, and intermediate-density lipoproteins, from general circulation. The oral administration of $A$. muciniphila also improved hepatic endoplasmic reticulum stress and metabolic inflammation in CREBH-null mice (Shen et al., 2016).

In a previous study, Sun et al. reported that berberine improved metabolic disorders caused by a high-fat diet and did so by regulating the GM-gut-brain axis. Berberine also increased the ratio of $\mathrm{B}: \mathrm{F}$ and the proportion of SCFAproducing bacteria, thus promoting the increased expression of glucagon-like peptide (GLP)-1 in intestinal L cells, a type of endocrine cell in the intestine that secretes GLP-1 (Sun et al., 2016). Other research reported a positive correlation between the abundance of bacteria belonging to the Akkermansia genus and the number of L-cells in the colon; the administration of $A$. muciniphila significantly increased GLP-1 release from colonic L-cells (Everard et al., 2013).

Zhang et al. also reported that berberine modulated the GM, enriched the population of SCFA-producing bacteria, and regulated microbial diversity, thus enhancing intestinal integrity (Zhang et al., 2015). These authors also revealed that Phascolarctobacterium, Anaerotruncus, and Oscillibacter, may be solely responsible for the beneficial effects of berberine on intestinal permeability. Berberine increased the expression of ZO-1 mRNA by inhibiting the abundance of Oscillibacter, thus antagonizing obesity.

Berberine can enrich the population of butyrate-producing bacteria in the GM (Wang et al., 2017b). Butyrate-induced upregulation of GLP-1 and PYY may be important in preventing or treating obesity and insulin resistance (Vidrine et al., 2014). Treatments with butyrate or increasing butyrate productions have been shown to prevent or attenuate obesity and insulin resistance (Li M. et al., 2016; Li X. et al., 2016; Goldsmith et al., 2017; Zhang et al., 2017). Butyrate has also been shown to increase B-adrenergic receptor profiles in adipocytes, which occurs via HDACi activity, a similar mechanism for upregulating fatty acid oxidation may occur in white adipose tissue (McNabney and Henagan, 2017). The HDACi activity of butyrate has also been associated with its ability to prevent adipose tissue inflammation, a contributing factor to insulin resistance during obesity (Ding et al., 2000; Wang et al., 2015). N-(1-carbamoyl-2-phenyl-ethyl) butyramide (FBA), a synthetic more palatable derivative of butyrate, in mice fed the HFD reduces hepatic fat accumulation and decreases metabolic/ mitochondrial efficiency, counteracting obesity, IR, and inflammation (Mollica et al., 2017). Elevation of SCFA availability by increasing dietary fiber intake or diet supplementation with butyrate may prevent the development of metabolic disarrangements and the insulin resistance associated with obesity (Galisteo et al., 2008; Nilsson et al., 2010).

\section{THE EFFECTS OF BERBERINE ON HYPERLIPIDEMIA}

Hyperlipidemia is a major component of the metabolic syndrome, and gives rise to increased levels of triglyceride (TG), total cholesterol (TC), and low-density lipoprotein cholesterol (LDL-C), while reducing the serum levels of highdensity lipoprotein cholesterol (HDL-C) (Chen et al., 2014). Berberine has been shown to exert a therapeutic effect on patients with hyperlipidemia (Kong et al., 2004).

The "one-drug-multiple-target" concept is characteristic of the treatment applied for hyperlipidemia. The main targets of berberine on the metabolism of lipids and glucose are IR and the LDLR. Interestingly, berberine has been shown to reduce the serum levels of lipids in humans, hamsters, rats, and mice (Kong et al., 2004; Chang et al., 2010; Wang et al., 2010). A clinical trial showed that berberine treatment reduced the levels of total cholesterol, triglycerides, and LDL cholesterol, while increasing the levels of HDL cholesterol after 3 months of treatment when compared with a placebo (Derosa et al., 2013).

In another study, the oral administration of berberine in 32 hypercholesterolemic patients for 3 months was shown to reduce serum levels of cholesterol by $29 \%$, triglycerides by $35 \%$ and LDL-cholesterol by 25\% (Kong et al., 2004). Furthermore, the treatment of hyperlipidemic hamsters with berberine reduced serum levels of cholesterol by $40 \%$ and LDL-cholesterol by $42 \%$, with a 3.5-fold increase in hepatic $L D L R$ mRNA expression and a 2.6-fold increase in hepatic LDLR protein levels (Kong et al., 2004; Yao et al., 2015). Berberine has also been shown to increase the abundance of Bacteroides, Parabacteroides, and Blautia genera, and eliminate or reduce the populations of Prevotella, Escherichia, Clostridium, and Sutterella genera in patients with hyperlipidemia (Li et al., 2016).

Berberine can change the abundance of intestinal mucus produced by $A$. muciniphila bacteria in an animal model for 
high-fat diets (Zhu et al., 2018). These results indicated that berberine can improve hyperlipidemia by affecting the composition of the intestinal flora. Wang et al. further reported that fecal nitrate reductase (NR) activity in patients with hyperlipidemia was higher than that in healthy individuals. The NR activity of intestinal bacteria plays a key role in promoting the intestinal absorption of berberine. In humans, individuals with high NR activity have been shown to exhibit higher levels of berberine in their blood compared with those with normal fecal NR activity, thereby suggesting a variation in the oral bioavailability of berberine (Wang et al., 2017c).

Other studies have shown that berberine can suppress the production of ATP and NADH levels by bacteria and also the levels of nicotinamide adenine dinucleotide. The ob/ob mice (C57BL/6J) were treated orally with berberine $(100 \mathrm{mg} / \mathrm{kg} /$ day $)$ for 10 days and their feces sample was taken for the bacterial composition analysis. Of the 50 genera, the abundance of 9 genera increased after berberine treatment. Seven of the nine genera were able to produce butyrate, including Enterobacter, Escherichia - Shigella, Incertae sedis, Lachnospiraceae FCS020 group, Akkermansia, Clostridium sensu stricto 1, and Bacteroides, with the biggest increase seen in Enterobacter and Escherichia -Shigella (Wang et al., 2017b). These actions resulted in increased levels of butyryl-CoA, thus promoting the GM to produce butyrate. Once released, butyrate enters the blood and is able to reduce the levels of lipids and glucose. However, the intraperitoneal administration of berberine was unable to increase the levels of butyrate but did reduce the levels of lipids and glucose in the blood. Therefore, berberine appears to act via two different models in order to reduce hyperlipidemia: firstly by exerting a direct effect via the circulation and secondly, by exerting an indirect effect via butyrate produced by GM (Wang et al., 2017b).

\section{THE EFFECTS OF BERBERINE ON LIVER DISEASE}

Previous research has shown that the liver is exposed to gut-derived bacterial metabolites and products (Eissa et al., 2018). A previous study showed that the concentrations and bioactive metabolites of berberine in the organs were higher than those in the blood during the progression of alcoholic liver disease (ALD) from steatohepatitis to fibrosis, cirrhosis, and then to end-stage liver disease. In addition, berberine was shown to be distributed rapidly in a range of tissues, but predominantly in the liver (Tan et al., 2013).

Berberine has also been shown to significantly reduce inflammation, fibrosis, and the levels of lipid peroxides in the liver (Zhang et al., 2016). The lipid-lowering effect of berberine occurs via a series of continuous events, including bile salt hydrolase (BSH) inhibition, significant increases in the levels of tauro-conjugated bile acids (especially TCA), and the activation of the FXR signaling pathway. These events reduce the expression levels of CD36 in the liver which then leads to a reduction in the hepatic uptake of long-chain fatty acids and the modulation of lipid metabolism in the liver (Sun et al., 2017).
Berberine activated a population with immune suppressive function, defined as granulocytic- myeloid-derived suppressor cell (G-MDSC)-like population, in the liver of mice with alleviating ALD. Berberine remarkably enhanced the increase of G-MDSC-like cells in blood and liver and decreased cytotoxic $\mathrm{T}$ cells correspondingly. Moreover, berberine changed the overall gut microbial community, primarily increased the abundance of A. muciniphila. Of note, depletion of gut microbiota abolished the inducing effect of berberine on G-MDSC-like population, and attenuated its hepatoprotective effect against alcohol in mice, suggesting intestinal flora might be involved in mediating the expansion of this protective population (Li S. et al., 2020). Patients with ALD have been shown to possess an increased abundance of endotoxin-producing Enterobacteriaceae, and a reduced abundance of SCFAs-producing bacteria, such as Lachnospiraceae and Ruminococcaceae. In a previous study, Grander et al. (2018) showed that A. muciniphila, a commensal type of bacteria, was associated with intestinal mucous layer in alcoholic hepatitis. These authors showed that clinical stool samples from patients with alcoholic hepatitis had the lowest relative abundance of A.muciniphila. Further experiments, using a mouse(C57BL/6J) model of ALD, reported improvements in alcohol-associated hepatic disease and intestinal barrier function following the administration of A. muciniphila (Grander et al., 2018). Other studies have shown that berberine can regulate SCFA-producing bacteria (Wang et al., 2017). Human and animal experiments in ALD and cirrhosis have further demonstrated that probiotics, including Lactobacillaceae spp. can improve the outcomes of these diseases (Han et al., 2015).

Primary biliary cholangitis (PBC) and primary sclerosing cholangitis (PSC) are both cholestatic liver disorders. Patients with $\mathrm{PBC}$ are known to possess reduced populations of beneficial bacteria such as Acidobacteria, Lachnobacterium, Bacteroides, and Ruminococcus, and increased populations of pathogens, such as proteobacteria, enterobacteriaceae, Veillonella, Streptococcus, and Klebsiella (Lv et al., 2016). Berberine is known to exhibit antibacterial activity against Streptococcus and Klebsiella (Zhou et al., 2016; Du et al., 2020).

The administration of berberine has been shown to restore the relative levels of bifidobacteria, along with the $\mathrm{B}: \mathrm{F}$ ratio, in mice $(\mathrm{BALB} / \mathrm{c})$ that were fed a high-fat diet. These changes subsequently led to an improvement in serum transaminase activity and non-alcoholic fatty liver disease activity scale score (Cao et al., 2016). Furthermore, the expression levels of CD14, IL1 , IL- 6 , and TNF- $\alpha$, were significantly downregulated in mice fed on a high-fat diet following berberine treatment (Cao et al., 2016). Berberine can also ameliorate intestinal dysbacteriosis and therefore reduce the liver toxicity caused by pathological/ pharmacological intervention. Berberine treatment was also shown to reduce the level of dextran sulfate sodium (DSS)induced intestinal dysbacteriosis and thus reduce acute liver toxicity (Qin et al., 2018). Therefore, the transplantation of fecal microbiota might represent a useful method to directly explore homeostatic alterations in the GM. Demethyleneberberine (DMB) is an essential in vivo metabolite of berberine. $\mathrm{DMB}$ has been 
shown to suppress the activation of hepatic stellate cells (HSCs) and induce apoptosis by regulating the nuclear factor-kappa $\mathrm{B}$ (NF- $\mathrm{KB})$ cascade. DMB also has inhibitory effects on collagen synthesis and is able to increase the degradatino of collagen by blocking the transforming growth factor $\beta 1$ (TGF- $\beta 1$ )-Smad signaling pathway. In addtion, DMB can reduce the expression of matrix metalloproteinases (MMPs) (Wang et al., 2016).

\section{THE EFFECTS OF BERBERINE ON DIABETES MELLITUS (DM)}

$\mathrm{DM}$ is a metabolic disease that is associated with high levels of morbidity and mortality. Alterations and in the GM, along with chronic systemic inflammation, have been shown to lead to DM (Sepp et al., 2014; Cui et al., 2018a). There is increasing evidence to show that changes in the GM are associated with IR and DM. In a previous study, Gao et al. proposed that the "bacteriamucosal immunity-inflammation-diabetes" axis can be utilized in the prevention and treatment of DM (Gao et al., 2017). Berberine may be efficacious against DM, and has been shown to exert its actions by modulating the GM (Zhang et al., 2015). In a previous study, Cui showed that the relative abundance of Bacteroidetes in the rat model of type II diabetes mellitus (T2DM) was lower than in normal rats; this difference was largely abolished following berberine treatment.

Research has shown that berberine fumarate (BF) may play a hypoglycemic role in rats with DM by regulating GM and metabolism. The administration of $\mathrm{BF}$ has been shown to significantly ameliorate metabolic disorders, and increase the populations of Bacteroidetes, Clostridia, Lactobacillales, Prevotellaceae, and Alloprevotella. In addition, the relative abundance of Clostridia in the rat intestine was negatively correlated with the host's blood glucose; BF treatment was shown to reduce the populations of Bacteroidales, Desulfovibrio, Lachnospiraceae, and Rikenellaceae in the rat model of T2DM. Moreover, BF has been shown to reduce inflammation, inhibit the overexpression of toll-like receptor (TLR) and phosphorylated c-Jun N-terminal kinase, and increase the expression of phosphoinositide 3-kinase, glucose transporter-2, and other proteins related to oxidative stress, thus promoting the glucose metabolism (Cui et al., 2018a).

Bacteria in the gut can decompose and metabolize berberine into dihydroberberine, thus preventing the absorption of disaccharides in the intestinal tract (Feng et al., 2015), and increasing the secretion of GLP-1 and GLP-2 to protect pancreatic islet cells and reduce the levels of glucose in the blood (Wei et al., 2014). Furthermore, berberine is able to regulate the expression of a range of related molecules in rats with DM, including the TLR4/MyD88/NF- $\mathrm{KB}$ signaling pathway (Gong et al., 2017). In the rat model of T2DM, the bioavailability of berberine is higher than that in normal rats. Furthermore, compared with berberine hydrochloride, berberine organic acid salts (especially BF and berberine succinate) can not only control the levels of blood sugar and avert the occurrence of hyperchloremia, but can also significantly improve the oral bioavailability of berberine (Cui et al., 2018b).
In particular, a number of observational studies have identified an association between elevated circulating levels of branchedchain amino acids (BCAAs) and poor metabolic health. In clinical studies, increased levels of BCAAs in the blood have been positively correlated with insulin resistance (Lynch and Adams, 2014). Berberine has been shown to reduce the relative abundance of BCAA-producing bacteria, including Clostridiales; the families of Streptococcaceae, Clostridiaceae, the Streptococcus genera, and Prevotella. Consequently, the increased serum levels of BCAAs induced by the consumption of a high-fat diet are reduced significantly following the administration of berberine. Furthermore, data from both healthy subjects and patients with DM indicate that berberine can improve glycemic control and modulate the circulating levels of BCAAs (Yue et al., 2019). Therefore, it is evident that the hypoglycemic effects of berberine may be related to improvements in the regulation of gut-derived hormones, the weakening of mechanisms in the intestinal mucosal, and the destruction of the immune-barrier.

Both berberine and metformin have been shown to cause changes in more than 20 genera in $\mathrm{db} / \mathrm{db}$ mice (C57BLKS/JNju, animal models of type 2 diabetes). Both of these treatments caused significant changes in the expression of seven OTUs, including increases in the prevalence of a range of SCFA-producing bacteria, including Butyricimonas, Coprococcus, and Ruminococcus (Zhang et al., 2019). Similar changes were observed in the content of SCFA-producing bacteria in the feces. Both of these treatments led to an increase in the populations of the symbiotic genera Lactobacillus and Akkermansia. In contrast, both treatments reduced the populations of Prevotella and Proteus, two types of opportunistic pathogens. Berberine and metformin were able to reduce weight gain and regulate the gut microbiome while suppressing intestinal inflammation and supporting the intestinal barrier (Zhang et al., 2019).

The combination of berberine and stachyose was previously shown to improve glycol-metabolism in T2DM mice (BKS-db) to a better extent than berberine alone (Li et al., 2020); this effect occurred by changes in the regulation of the intestinal microbiota and fecal metabolomics. Following treatment with berberine and stachyose, there was a reduction in the abundance of Saccharibacteria, Deferribacteres, Actinobacteria, and Firmicutes, but an increase in the abundance of Verrucomicrobia. Furthermore, compared with berberine treatment alone, there was a significant increase in the abundance of Verrucomicrobia when stachyose and berberine were administered in combination ( $\mathrm{Li}$ et al., 2020).

\section{THE EFFECTS OF BERBERINE ON CANCER}

Colorectal cancer (CRC) is the third most commonly encountered malignant tumor and the fourth leading cause of cancer mortality in the world (Siegel et al., 2019). In 2019, approximately 145,600 new cases of CRC, and 51,020 deaths, were estimated to have involved CRC (Song et al., 2020). An increasing body of data now support the fact that changes in the intestinal microbiome allow environmental risk factors to initiate 
and promote CRC (Song and Chan, 2019). Previous research has shown that berberine can inhibit the development of colorectal cancer (CRC) (Habtemariam, 2016).

A. muciniphila is a gram-negative anaerobic bacterium that is selectively reduced in the fecal microbiota of patients with colitis or colitis-associated cancer (CAC). amuc_1100 is a special protein that can be isolated from the outer membrane of $A$. muciniphila. Once isolated, amuc_1100 still exerts biological activity and plays a beneficial role at the temperature used for pasteurization. A. muciniphila or amuc_1100 has been shown to alleviate colitis and CAC, reduce $\mathrm{CD}^{+}$cytotoxic T lymphocytes (cTls), and the infiltration of macrophages in the colon, and may therefore represent a promising therapeutic target for the treatment of colitis and CRC (Wang et al., 2020). However, research has shown that the population of Akkermansia was significantly increased in a mouse model (BALB/c) of CAC fed a high-fat diet ( $\mathrm{Wu}$ et al., 2016). The $\mathrm{Apc}^{\mathrm{min} /+}$ mouse model (C57BL/6J) has a tumorigenic phenotype and can develop intestinal tumors; research has shown that high-fat diet could accelerate the process of carcinogenesis. Berberine has been shown to significantly reduce intestinal-tumor development and cause changes in the structure of the GM in $\mathrm{Apc}^{\mathrm{min} /+}$ mice (C57BL/6J) fed on a high-fat diet (Wang et al., 2018). Berberine can clearly inhibit the increased abundance of Verrucomicrobia at the phylum level. At the genus level, berberine can suppress Akkermansia and increase the abundance of some SCFAproducing bacteria (Wang et al., 2018).

Berberine has also been shown to promote the interaction between retinoid $\mathrm{X}$ receptor alpha $(\mathrm{RXR} \alpha)$ and nuclear $\beta$ catenin; this leads to the Casitas B-lineage lymphoma (c-Cbl)mediated degradation of $\beta$-catenin, thereby inhibiting the proliferation of colon cancer cells (Ruan et al., 2017).

\section{THE EFFECTS OF BERBERINE ON OTHER DISEASES}

The modulation of berberine-induced GM plays a significant role in the development of IBD and atherosclerosis (Cui et al., 2018). IBD is caused by dysregulation of the immune responses in the intestinal mucosal in hosts that are genetically susceptible (Strober et al., 2007). Berberine has also been shown to inhibit the production of pro-inflammatory cytokines in colonic macrophages and epithelial cells, and promote apoptosis in the colon macrophages of mice $(\mathrm{C} 57 \mathrm{BL} / 6)$ treated with DSS. Berberine was also shown to reduce the activation of the signaling pathways that produce proinflammatory cytokines (including mitogen-activated protein kinase and NF- $\mathrm{KB}$ ) in colonic macrophages and epithelial cells in DSS-treated mice (Yan et al., 2012). In the intestinal mucositis induced by 5 -fluorouracil (5-Fu) using rat model, berberine significantly increased the levels of butyrate and glutamine in feces from $5-\mathrm{Fu}$ treated rats. In terms of gut microbiota, berberine enriched the relative abundance of Firmicutes and decreased Proteobacteria at the phylum level. Meanwhile, berberine increased the proportion of unclassified $f_{-}$Porphyromonadaceae,
unclassified_f_Lachnospiraceae, Lactobacillus, unclassified_ o_Clostridiales, Ruminococcus, Prevotella, Clostridium IV, and decreased Escherichia/Shigella at the genera level (Chen et al., 2020).

Clinical evidence suggests that berberine can reduce endothelial inflammation and improve vascular health (Cicero and Baggioni, 2016). Shi et al. further reported that berberine may modulate the composition of the GM in subjects with atherosclerosis (Shi et al., 2018). Other studies have shown that berberine could be used to treat atherosclerosis by increasing the abundance of Akkermansia spp in mice(C57BL) fed a high-fat diet (Zhu et al., 2018). In addition, berberine was shown to reduce HFD-induced metabolic endotoxemia and the expression of proinflammatory cytokines and chemokines in the arteries and in the intestine.

Other research has shown that berberine can reduce the expression of hepatic flavin-containing monooxygenase 3 (FMO3) and the serum levels of proteins involved in the trimethylamine N-oxide FXR signaling pathway (Shi et al., 2018). Similarly, the levels of primary bile acids (e.g., $\beta$ muricholic acid and tauroursodeoxycholic acid) were shown to be increased in the livers and sera of mice (C57BL/6) fed berberine; the levels of secondary bile acids (lithocholic acid and T-conjugates) were reduced (Guo et al., 2016). Another study reported that the expression of bile acid-synthetic enzymes (e.g., cytochrome P450 (Cyp)7a1 and Cyp8b1), and an uptake transporter sodium taurocholate co-transporting polypeptide (Ntcp), increased by 39 to $400 \%$ in the livers of mice fed high doses of berberine; however, there was no significant change in the expression levels of the nuclear receptor and efflux transporter (Guo et al., 2016).

Berberine treatment has also been shown to increase the abundance of Akkermansia in the intestine and alleviate atherosclerosis in Apoe (-/-) mice fed a high-fat diet (Zhu et al., 2018). Collectively, these data indicate that berberine may play different regulatory roles in different disease models and that berberine acts via many different systems on a range of targets in the treatment of disease.

\section{CONCLUSIONS}

Berberine has various pharmacologic properties and multispectrum therapeutic applications (Imenshahidi and Hosseinzadeh, 2019). The GM is an important environmental factor that interacts with its host, and participates in the occurrence and development of various diseases. Several clinical studies have shown that berberine can be used in treatment of different diseases (e.g., DM, hyperlipidemia, cancer, metabolic syndrome, polycystic ovarian syndrome, liver disease) by GM regulation (Figure 1).

This review mainly elucidates the role of berberine in a variety of diseases by regulating intestinal flora. However, three main factors need to be addressed. First, there are genetic differences between rodents and humans, so the regulatory effect of the GM through berberine must be demonstrated clinically. Second, 


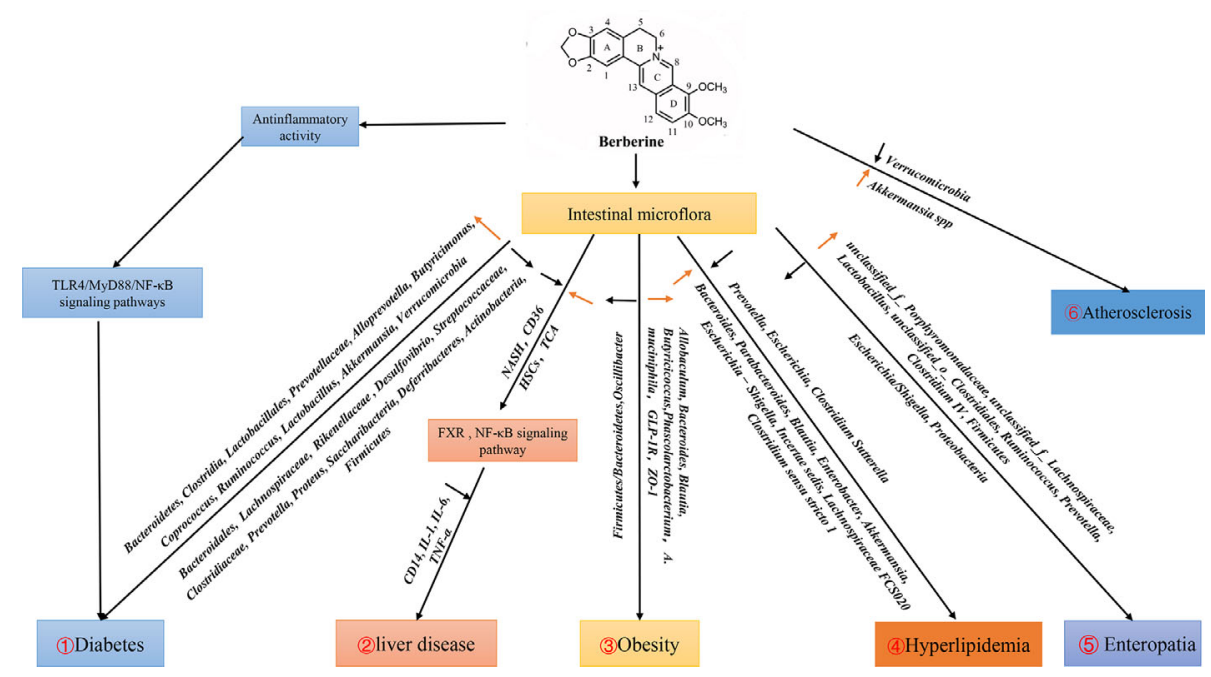

FIGURE 1 | Effects of berberine on diseases by regulating intestinal flora. 1. Berberine can activate TLR4/MyD88/NF- $\mathrm{BB}$ signal pathway to play an anti-inflammatory role, and increase the populations of Bacteroidetes, Clostridia, Lactobacillales, Prevotellaceae, Alloprevotella, Butyricimonas, Coprococcus, Ruminococcus, Lactobacillus, Akkermansia, Verrucomicrobia, reduce the populations of Bacteroidales, Lachnospiraceae, Rikenellaceae, Desulfovibrio, Streptococcaceae, Clostridiaceae, Prevotella, Proteus, Saccharibacteria, Deferribacteres, Actinobacteria, which affect the development of diabetes. 2. Berberine affects liver diseases by regulating FXR and NF-кB signaling pathways through intestinal flora. 3. Berberine down-regulate F:B (Firmicutes : Bacteroidetes) and up-regulate SCFA-producing bacteria Allobaculum, Bacteroides, Blautia, Butyricicoccus, Phascolarctobacterium, A. muciniphila and GLP-1R in obesity. 4. Berberine regulates hyperlipidemia by reducing Prevotella, Escherichia, Clostridium Sutterella and increase Bacteroides, Parabacteroides, Blautia, Enterobacter, Akkermansia, Escherichia-Shigella, Incertae sedis, Lachnospiraceae FCS020, Clostridium sensu stricto 1. 5. In Enteropatia, berberine enriched the relative abundance of Firmicutes and decreased Proteobacteria at the phylum level. Meanwhile, berberine increased the propotion of unclassified_f_Porphyromonadaceae, unclassified_f_Lachnospiraceae, Lactobacillus, unclassified_o_Clostridiales, Ruminococcus, Prevotella, Clostridium IV, and decreased Escherichia/Shigella at the genera level. 6. Berberine affect the

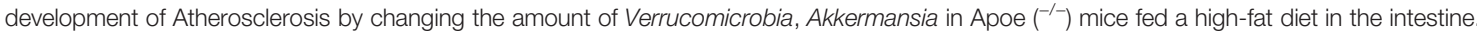

better understanding of how the GM regulates glucose metabolism and DM complications is needed. Third, the US Food and Drug Administration has not approved berberine for any indication.

\section{AUTHOR CONTRIBUTIONS}

This manuscript was designed by LW and XS and drafted by LZ, XW, and RY. FC, YL, ZZ, and ZW contributed to writing. All authors contributed to the article and approved the submitted version.

\section{REFERENCES}

Caliceti, C., Franco, P., Spinozzi, S., Roda, A., and Cicero, A. F. (2016). Berberine: New Insights from Pharmacological Aspects to Clinical Evidences in the Management of Metabolic Disorders. Curr. Med. Chem. 23 (14), 1460-1476. doi: 10.2174/0929867323666160411143314

Cao, Y., Pan, Q., Cai, W., Shen, F., Chen, G. Y., Xu, L. M., et al. (2016). Modulation of Gut Microbiota by Berberine Improves Steatohepatitis in High-Fat Diet-Fed BALB/C Mice. Arch. Iran Med. 19 (3), 197-203. doi: 0161903/AIM.008

Chang, X., Yan, H., Fei, J., Jiang, M., Zhu, H., Lu, D., et al. (2010). Berberine reduces methylation of the MTTP promoter and alleviates fatty liver induced by a high-fat diet in rats. J. Lipid Res. 51 (9), 2504-2515. doi: 10.1194/jlr.M001958

Chen, H., Miao, H., Feng, Y. L., Zhao, Y. Y., and Lin, R. C. (2014). Metabolomics in dyslipidemia. Adv. Clin. Chem. 66, 101-119. doi: 10.1016/b978-0-12-801401$1.00004-9$

\section{FUNDING}

This work was supported by National Natural Science Foundation of China (No. 81902081 and 81871682), China Postdoctoral Science Foundation (No. 2018M640858, 2019T120771), the Natural Science Foundation of Guangdong Province (No. 2020A1515011573 and 2019A1515012068), Pearl River Nova Program of Guangzhou (Grant No.201710010030), Fundamental Research Funds for the Central Universities (No. 19ykpy170, 17ykpy09, No.19ykpy29), National Science and Technology Major Project (No. 2018ZX10101002-001).

Chen, X., Zhang, Y., Zhu, Z., Liu, H., Guo, H., Xiong, C., et al. (2016). Protective effect of berberine on doxorubicininduced acute hepatorenal toxicity in rats. Mol. Med. Rep. 13 (5), 3953-3960. doi: 10.3892/mmr.2016.5017

Chen, H., Zhang, F., Li, R., Liu, Y., Wang, X., Zhang, X., et al. (2020). Berberine regulates fecal metabolites to ameliorate 5-fluorouracil induced intestinal mucositis through modulating gut microbiota. Biomed. Pharmacother. = Biome. Pharmacother. 124:109829. doi: 10.1016/j.biopha.2020.109829

Cicero, A. F., and Baggioni, A. (2016). Berberine and Its Role in Chronic Disease. Adv. Exp. Med. Biol. 928, 27-45. doi: 10.1007/978-3-319-41334-1_2

Cok, A., Plaisier, C., Salie, M. J., Oram, D. S., Chenge, J., and Louters, L. L. (2011). Berberine acutely activates the glucose transport activity of GLUT1. Biochimie 93 (7), 1187-1192. doi: 10.1016/j.biochi.2011.04.013

Cui, H., Cai, Y., Wang, L., Jia, B., Li, J., Zhao, S., et al. (2018). Berberine Regulates Treg/ Th17 Balance to Treat Ulcerative Colitis Through Modulating the Gut Microbiota in the Colon. Front. Pharmacol. 9 (1), 103. doi: 10.3389/fphar.2018.00571 
Cui, H. X., Hu, Y. N., Li, J. W., and Yuan, K. (2018a). Hypoglycemic Mechanism of the Berberine Organic Acid Salt under the Synergistic Effect of Intestinal Flora and Oxidative Stress. Oxid. Med. Cell Longev. 2018, 8930374. doi: 10.1155/2018/8930374

Cui, H. X., Hu, Y. N., Li, J. W., Yuan, K., and Guo, Y. (2018b). Preparation and Evaluation of Antidiabetic Agents of Berberine Organic Acid Salts for Enhancing the Bioavailability. Molecules 24 (1), 103. doi: 10.3390/molecules 24010103

Derosa, G., D’Angelo, A., Bonaventura, A., Bianchi, L., Romano, D., and Maffioli, P. (2013). Effects of berberine on lipid profile in subjects with low cardiovascular risk. Expert Opin. Biol. Ther. 13 (4), 475-482. doi: 10.1517/14712598.2013.776037

Ding, S. T., Smith, E. O., McNeel, R. L., and Mersmann, H. J. (2000). Modulation of porcine adipocyte beta-adrenergic receptors by hormones and butyrate. J. Anim. Sci. 78 (4), 927-933. doi: 10.2527/2000.784927x

Du, G. F., Le, Y. J., Sun, X., Yang, X. Y., and He, Q. Y. (2020). Proteomic investigation into the action mechanism of berberine against Streptococcus pyogenes. J. Proteomics 215:103666. doi: 10.1016/j.jprot.2020.103666

Eissa, L. A., Kenawy, H.II, El-Karef, A., Elsherbiny, N. M., and El-Mihi, K. A. (2018). Antioxidant and anti-inflammatory activities of berberine attenuate hepatic fibrosis induced by thioacetamide injection in rats. Chem. Biol. Interact. 294, 91-100. doi: 10.1016/j.cbi.2018.08.016

Everard, A., Belzer, C., Geurts, L., Ouwerkerk, J. P., Druart, C., Bindels, L. B., et al. (2013). Cross-talk between Akkermansia muciniphila and intestinal epithelium controls diet-induced obesity. Proc. Natl. Acad. Sci. United States America 110 (22), 9066-9071. doi: 10.1073/pnas.1219451110

Feng, R., Shou, J. W., Zhao, Z. X., He, C. Y., Ma, C., Huang, M., et al. (2015). Transforming berberine into its intestine-absorbable form by the gut microbiota. Sci. Rep. 5:12155. doi: 10.1038/srep12155

Galisteo, M., Duarte, J., and Zarzuelo, A. (2008). Effects of dietary fibers on disturbances clustered in the metabolic syndrome. J. Nutr. Biochem. 19 (2), 7184. doi: 10.1016/j.jnutbio.2007.02.009

Gao, Z., Li, Q., Wu, X., Zhao, X., Zhao, L., and Tong, X. (2017). New Insights into the Mechanisms of Chinese Herbal Products on Diabetes: A Focus on the "Bacteria-Mucosal Immunity-Inflammation-Diabetes" Axis. J. Immunol. Res. 2017:1813086. doi: 10.1155/2017/1813086

Goldsmith, F., Guice, J., Page, R., Welsh, D. A., Taylor, C. M., Blanchard, E. E., et al. (2017). Obese ZDF rats fermented resistant starch with effects on gut microbiota but no reduction in abdominal fat. Mol. Nutr. Food Res. 61 (1), 10.1002/mnfr.201501025. doi: 10.1002/mnfr.201501025.doi.org/10.1002/ mnfr. 201501025

Gong, J., Hu, M., Huang, Z., Fang, K., Wang, D., Chen, Q., et al. (2017). Berberine Attenuates Intestinal Mucosal Barrier Dysfunction in Type 2 Diabetic Rats. Front. Pharmacol. 8:42:42. doi: 10.3389/fphar.2017.00042

Grander, C., Adolph, T. E., Wieser, V., Lowe, P., Wrzosek, L., Gyongyosi, B., et al. (2018). Recovery of ethanol-induced Akkermansia muciniphila depletion ameliorates alcoholic liver disease. Gut 67 (5), 891-901. doi: 10.1136/gutjnl-2016-313432

Guo, Y., Zhang, Y., Huang, W., Selwyn, F. P., and Klaassen, C. D. (2016). Doseresponse effect of berberine on bile acid profile and gut microbiota in mice. BMC Complement. Altern. Med. 16 (1), 394. doi: 10.1186/s12906-016-1367-7

Habtemariam, S. (2016). Berberine and inflammatory bowel disease: A concise review. Pharmacol. Res. 113 (Pt A), 592-599. doi: 10.1016/j.phrs.2016.09.041

Han, S. H., Suk, K. T., Kim, D. J., Kim, M. Y., Baik, S. K., Kim, Y. D., et al. (2015). Effects of probiotics (cultured Lactobacillus subtilis/Streptococcus faecium) in the treatment of alcoholic hepatitis: randomized-controlled multicenter study. Eur. J. Gastroenterol. Hepatol. 27 (11), 1300-1306. doi: 10.1097/MEG.0000000000000458

Hasanein, P., Ghafari-Vahed, M., and Khodadadi, I. (2017). Effects of isoquinoline alkaloid berberine on lipid peroxidation, antioxidant defense system, and liver damage induced by lead acetate in rats. Redox Rep. 22 (1), 42-50. doi: 10.1080/ 13510002.2016.1140406

Imenshahidi, M., and Hosseinzadeh, H. (2019). Berberine and barberry (Berberis vulgaris): A clinical review. Phytother. Res. 33 (3), 504-523. doi: 10.1002/ptr.6252

Jin, Y., Khadka, D. B., and Cho, W. J. (2016). Pharmacological effects of berberine and its derivatives: a patent update. Expert Opin. Ther. Pat. 26 (2), 229-243. doi: $10.1517 / 13543776.2016$

Kamada, N., Seo, S. U., Chen, G. Y., and Nunez, G. (2013). Role of the gut microbiota in immunity and inflammatory disease. Nat. Rev. Immunol. 13 (5), 321-335. doi: 10.1038/nri3430

Kong, W., Wei, J., Abidi, P., Lin, M., Inaba, S., Li, C., et al. (2004). Berberine is a novel cholesterol-lowering drug working through a unique mechanism distinct from statins. Nat. Med. 10 (12), 1344-1351. doi: 10.1038/nm1135
Kuno, T., Hirayama-Kurogi, M., Ito, S., and Ohtsuki, S. (2016). Effect of Intestinal Flora on Protein Expression of Drug-Metabolizing Enzymes and Transporters in the Liver and Kidney of Germ-Free and Antibiotics-Treated Mice. Mol. Pharm. 13 (8), 2691-2701. doi: 10.1021/acs.molpharmaceut.6b00259

Leal-Díaz, A. M., Noriega, L. G., Torre-Villalvazo, I., Torres, N., AlemánEscondrillas, G., López-Romero, P., et al. (2016). Aguamiel concentrate from Agave salmiana and its extracted saponins attenuated obesity and hepatic steatosis and increased Akkermansia muciniphila in C57BL6 mice. Sci. Rep. 6, 34242. doi: 10.1038/srep34242

Li, C. N., Wang, X., Lei, L., Liu, M. Z., Li, R. C., Sun, S. J., et al. (2020). Berberine combined with stachyose induces better glycometabolism than berberine alone through modulating gut microbiota and fecal metabolomics in diabetic mice. Phytotherapy Res. PTR 34 (5), 1166-1174. doi: 10.1002/ptr.6588

Li, M., Shu, X., Xu, H., Zhang, C., Yang, L., Zhang, L., et al. (2016). Integrative analysis of metabolome and gut microbiota in diet-induced hyperlipidemic rats treated with berberine compounds. J. Transl. Med. 14 (1), 237. doi: 10.1186/ s12967-016-0987-5

Li, S., Wang, N., Tan, H. Y., Chueng, F., Zhang, Z. J., Yuen, M. F., et al. (2020). Modulation of gut microbiota mediates berberine-induced expansion of immuno-suppressive cells to against alcoholic liver disease. Clin. Trans. Med. 10 (4), e112. doi: $10.1002 / \mathrm{ctm} 2.112$

Li, X., Xu, Q., Jiang, T., Fang, S., Wang, G., Zhao, J., et al. (2016). A comparative study of the antidiabetic effects exerted by live and dead multi-strain probiotics in the type 2 diabetes model of mice. Food Funct. 7 (12), 4851-4860. doi: $10.1039 / \mathrm{c} 6$ fo0 $1147 \mathrm{k}$

Louis, P., Hold, G. L., and Flint, H. J. (2014). The gut microbiota, bacterial metabolites and colorectal cancer. Nat. Rev. Microbiol. 12 (10), 661-672. doi: $10.1038 /$ nrmicro3344

Lv, L. X., Fang, D. Q., Shi, D., Chen, D. Y., Yan, R., Zhu, Y. X., et al. (2016). Alterations and correlations of the gut microbiome, metabolism and immunity in patients with primary biliary cirrhosis. Environ. Microbiol. 18 (7), 22722286. doi: $10.1111 / 1462-2920.13401$

Lynch, C. J., and Adams, S. H. (2014). Branched-chain amino acids in metabolic signalling and insulin resistance. Nat. Rev. Endocrinol. 10 (12), 723-736. doi: 10.1038/nrendo.2014

Maslowski, K. M., Vieira, A. T., Ng, A., Kranich, J., Sierro, F., Yu, D., et al. (2009). Regulation of inflammatory responses by gut microbiota and chemoattractant receptor GPR43. Nature 461 (7268), 1282-1286. doi: 10.1038/nature08530

McNabney, S. M., and Henagan, T. M. (2017). Short Chain Fatty Acids in the Colon and Peripheral Tissues: A Focus on Butyrate, Colon Cancer, Obesity and Insulin Resistance. Nutrients 9 (12), 1348. doi: 10.3390/nu9121348

Mollica, M. P., Mattace Raso, G., Cavaliere, G., Trinchese, G., De Filippo, C., Aceto, S., et al. (2017). Butyrate regulates liver mitochondrial function, efficiency, and dynamics in insulin-resistant obese mice. Diabetes 66 (5), 1405-1418. doi: 10.2337/db16-0924

Nilsson, A. C., Östman, E. M., Knudsen, K. E., Holst, J. J., and Björck, I. M. (2010). A cereal-based evening meal rich in indigestible carbohydrates increases plasma butyrate the next morning. J. Nutr. 140 (11), 1932-1936. doi: $10.3945 /$ in. 110.123604

Qin, C., Zhang, H., Zhao, L., Zeng, M., Huang, W., Fu, G., et al. (2018). Microbiota transplantation reveals beneficial impact of berberine on hepatotoxicity by improving gut homeostasis. Sci. China Life Sci. 61 (12), 1537-1544. doi: 10.1007/s11427-017-9202-0

Roediger, W. E. (1982). Utilization of nutrients by isolated epithelial cells of the rat colon. Gastroenterology 83 (2), 424-429.

Roudini, L., NayebZadeh Eidgahi, N., Rahimi, H. R., Saberi, M. R., Amiri Tehranizadeh, Z., Beigoli, S., et al. (2019). Determining the interaction behavior of calf thymus DNA with berberine hydrochloride in the presence of linker histone: a biophysical study. J. Biomol. Struct. Dyn. 38 (2), 364-381. doi: $10.1080 / 07391102.2019$

Round, J. L., and Mazmanian, S. K. (2009). The gut microbiota shapes intestinal immune responses during health and disease. Nat. Rev. Immunol. 9 (5), 313323. doi: $10.1038 /$ nri2515

Ruan, H., Zhan, Y. Y., Hou, J., Xu, B., Chen, B., Tian, Y., et al. (2017). Berberine binds RXRalpha to suppress beta-catenin signaling in colon cancer cells. Oncogene 36 (50), 6906-6918. doi: 10.1038/onc.2017.296

Schneeberger, M., Everard, A., Gómez-Valadés, A. G., Matamoros, S., Ramírez, S. Delzenne, N. M., et al. (2015). Akkermansia muciniphila inversely correlates with 
the onset of inflammation, altered adipose tissue metabolism and metabolic disorders during obesity in mice. Sci. Rep. 5, 16643. doi: 10.1038/srep16643

Sekirov, I., Russell, S. L., Antunes, L. C., and Finlay, B. B. (2010). Gut microbiota in health and disease. Physiol. Rev. 90 (3), 859-904. doi: 10.1152/physrev.00045.2009

Sepp, E., Kolk, H., Loivukene, K., and Mikelsaar, M. (2014). Higher blood glucose level associated with body mass index and gut microbiota in elderly people. Microb. Ecol. Health Dis. 25, 10.3402/mehd.v25.22857. doi: 10.3402/ mehd.v25.22857

Shen, J., Tong, X., Sud, N., Khound, R., Song, Y., Maldonado-Gomez, M. X., et al. (2016). Low-Density Lipoprotein Receptor Signaling Mediates the Triglyceride-Lowering Action of Akkermansia muciniphila in GeneticInduced Hyperlipidemia. Arterioscler. Thromb. Vasc. Biol. 36 (7), 1448-1456. doi: 10.1161/ATVBAHA.116.307597

Shi, Y., Hu, J., Geng, J., Hu, T., Wang, B., Yan, W., et al. (2018). Berberine treatment reduces atherosclerosis by mediating gut microbiota in Aope-/- mice. BioMed. Pharmacother. 107, 1556-1563. doi: 10.1016/j.biopha.2018.08.148

Siegel, R. L., Miller, K. D., and Jemal, A. (2019). Cancer statistics.(2019). CA Cancer J. Clin. 69 (1), 7-34. doi: 10.3322/caac.21551

Song, M., and Chan, A. T. (2019). Environmental Factors, Gut Microbiota, and Colorectal Cancer Prevention. Clin. Gastroenterol. Hepatol. Off. Clin. Pract. J. Am. Gastroenterol. Assoc. 17 (2), 275-289. doi: 10.1016/j.cgh.2018.07.012

Song, M., Chan, A. T., and Sun, J. (2020). Influence of the Gut Microbiome, Diet, and Environment on Risk of Colorectal Cancer. Gastroenterology 158 (2), 322340. doi: 10.1053/j.gastro.2019.06.048

Strober, W., Fuss, I., and Mannon, P. (2007). The fundamental basis of inflammatory bowel disease. J. Clin. Invest. 117 (3), 514-521. doi: 10.1172/JCI30587

Sun, H., Wang, N., Cang, Z., Zhu, C., Zhao, L., Nie, X., et al. (2016). Modulation of Microbiota-Gut-Brain Axis by Berberine Resulting in Improved Metabolic Status in High-Fat Diet-Fed Rats. Obes. Facts 9 (6), 365-378. doi: 10.1159/000449507

Sun, R., Yang, N., Kong, B., Cao, B., Feng, D., Yu, X., et al. (2017). Orally Administered Berberine Modulates Hepatic Lipid Metabolism by Altering Microbial Bile Acid Metabolism and the Intestinal FXR Signaling Pathway. Mol. Pharmacol. 91 (2), 110-122. doi: 10.1124/mol.116.106617

Tan, X. S., Ma, J. Y., Feng, R., Ma, C., Chen, W. J., Sun, Y. P., et al. (2013). Tissue distribution of berberine and its metabolites after oral administration in rats. PloS One 8 (10), e77969. doi: 10.1371/journal.pone.0077969

Tian, Y., Cai, J., Gui, W., Nichols, R. G., Koo, I., Zhang, J., et al. (2019). Berberine Directly Affects the Gut Microbiota to Promote Intestinal Farnesoid X Receptor Activation. Drug Metab. Dispos. 47 (2), 86-93. doi: 10.1124/dmd.118.083691

Turnbaugh, P. J., Ley, R. E., Mahowald, M. A., Magrini, V., Mardis, E. R., and Gordon, J.II (2006). An obesity-associated gut microbiome with increased capacity for energy harvest. Nature 444 (7122), 1027-1031. doi: 10.1038/nature05414

Vidrine, K., Ye, J., Martin, R. J., McCutcheon, K. L., Raggio, A. M., Pelkman, C., et al. (2014). Resistant starch from high amylose maize (HAM-RS2) and dietary butyrate reduce abdominal fat by a different apparent mechanism. Obes. (Silver Spring Md.) 22 (2), 344-348. doi: 10.1002/oby.20501

Walker, A. W., and Parkhill, J. (2013). Microbiology. Fighting Obes. Bacteria Sci. 341 (6150), 1069-1070. doi: 10.1126/science.1243787

Wang, Y., Jia, X., Ghanam, K., Beaurepaire, C., Zidichouski, J., and Miller, L. (2010). Berberine and plant stanols synergistically inhibit cholesterol absorption in hamsters. Atherosclerosis 209 (1), 111-117. doi: 10.1016/j.atherosclerosis.2009.08.050

Wang, X., He, G., Peng, Y., Zhong, W., Wang, Y., and Zhang, B. (2015). Sodium butyrate alleviates adipocyte inflammation by inhibiting NLRP3 pathway. Sci. Rep. 5:12676. doi: 10.1038/srep12676

Wang, Y., Zhao, Z., Yan, Y., Qiang, X., Zhou, C., Li, R., et al. (2016). Demethyleneberberine Protects against Hepatic Fibrosis in Mice by Modulating NF-kappaB Signaling. Int. J. Mol. Sci. 17 (7), 1036. doi: 10.3390/ijms17071036

Wang, L. L., Guo, H. H., Huang, S., Feng, C. L., Han, Y. X., and Jiang, J. D. (2017). Comprehensive evaluation of SCFA production in the intestinal bacteria regulated by berberine using gas-chromatography combined with polymerase chain reaction. J. Chromatogr. B Anal. Technol. Biomed. Life Sci. 1057, 70-801057:70-80. doi: 10.1016/j.jchromb.2017.05.004 Sci. 2017 Jul 1.

Wang, K., Feng, X., Chai, L., Cao, S., and Qiu, F. (2017a). The metabolism of berberine and its contribution to the pharmacological effects. Drug Metab. Rev. 49 (2), 139-157. doi: 10.1080/03602532.2017.1306544

Wang, Y., Shou, J. W., Li, X. Y., Zhao, Z. X., Fu, J., He, C. Y., et al. (2017b). Berberine-induced bioactive metabolites of the gut microbiota improve energy metabolism. Metabolism 70, 72-84. doi: 10.1016/j.metabol.2017.02.003
Wang, Y., Tong, Q., Shou, J. W., Zhao, Z. X., Li, X. Y., Zhang, X. F., et al. (2017c). Gut Microbiota-Mediated Personalized Treatment of Hyperlipidemia Using Berberine. Theranostics 7 (9), 2443-2451. doi: 10.7150/thno.18290

Wang, H., Guan, L., Li, J., Lai, M., and Wen, X. (2018). The Effects of Berberine on the Gut Microbiota in Apc (min/+) Mice Fed with a High Fat Diet. Molecules 23 (9), 2298. doi: 10.3390/molecules23092298

Wang, L., Tang, L., Feng, Y., Zhao, S., Han, M., Zhang, C., et al. (2020). A purified membrane protein from akkermansia muciniphila or the pasteurised bacterium blunts colitis associated tumourigenesis by modulation of $\mathrm{cd} 8+\mathrm{t}$ cells in mice. Gut 69 (11), 1988-1997. doi: 10.1136/gutjnl-2019-320105

Wei, S. C., Dong, S., Xu, L. J., and Zhang, C. Y. (2014). Intestinal absorption of berberine and 8-hydroxy dihydroberberine and their effects on sugar absorption in rat small intestine. J. Huazhong Univ. Sci. Technolog. Med. Sci. 34 (2), 186-189. doi: 10.1007/s11596-014-1256-6

Wu, M., Wu, Y., Deng, B., Li, J., and Zhong, G. (2016). Isoliquiritigenin decreases the incidence of colitis-associated colorectal cancer by modulating the intestinal microbiota. Oncotarget 7 (51), 85318. doi: 10.18632/oncotarget.13347

Yan, F., Wang, L., Shi, Y., Cao, H., Liu, L., Washington, M. K., et al. (2012). Berberine promotes recovery of colitis and inhibits inflammatory responses in colonic macrophages and epithelial cells in DSS-treated mice. Am. J. Physiol. Gastrointest. Liver Physiol. 302 (5), G504-G514. doi: 10.1152/ajpgi.00312.2011

Yang, Y., Kang, N., Xia, H., Li, J., Chen, L., and Qiu, F. (2010). Metabolites of protoberberine alkaloids in human urine following oral administration of Coptidis Rhizoma decoction. Planta. Med. 76 (16), 1859-1863. doi: 10.1055/s-0030-1250053

Yao, J., Kong, W., and Jiang, J. (2015). Learning from berberine: Treating chronic diseases through multiple targets. Sci. China Life Sci. 58 (9), 854-859. doi: 10.1007/s11427-013-4568-z

Yue, S. J., Liu, J., Wang, A. T., Meng, X. T., Yang, Z. R., Peng, C., et al. (2019). Berberine alleviates insulin resistance by reducing peripheral branched-chain am ino acids. Am. J. Physiol. Endocrinol. Metab. 2019 Jan 1;316 (1), E73-E85. doi: 10.1152/ajpendo.00256.2018

Zhang, X., Zhao, Y., Zhang, M., Pang, X., Xu, J., Kang, C., et al. (2012). Structural changes of gut microbiota during berberine-mediated prevention of obesity and insulin resistance in high-fat diet-fed rats. PloS One 7 (8), e42529. doi: 10.1371/journal.pone.0042529

Zhang, X., Zhao, Y., Xu, J., Xue, Z., Zhang, M., Pang, X., et al. (2015). Modulation of gut microbiota by berberine and metformin during the treatment of high-fat diet-induced obesity in rats. Sci. Rep. 5:14405. doi: 10.1038/srep14405

Zhang, Z., Li, B., Meng, X., Yao, S., Jin, L., Yang, J., et al. (2016). Berberine prevents progression from hepatic steatosis to steatohepatitis and fibrosis by reducing endoplasmic reticulum stress. Sci. Rep. 6:20848. doi: 10.1038/srep20848

Zhang, L., Du, J., Yano, N., Wang, H., Zhao, Y. T., Dubielecka, P. M., et al. (2017). Sodium Butyrate Protects -Against High Fat Diet-Induced Cardiac Dysfunction and Metabolic Disorders in Type II Diabetic Mice. J. Cell. Biochem. 118 (8), 2395-2408. doi: 10.1002/jcb.25902

Zhang, W., Xu, J. H., Yu, T., and Chen, Q. K. (2019). Effects of berberine and metformin on intestinal inflammation and gut microbiome composition in $\mathrm{db} / \mathrm{db}$ mice. Biomed. Pharmacother.$=$ Biome. Pharmacother. $118: 109131$. doi: 10.1016/j.biopha.2019.109131

Zhou, X. Y., Ye, X. G., He, L. T., Zhang, S. R., Wang, R. L., Zhou, J., et al. (2016). In vitro characterization and inhibition of the interaction between ciprofloxacin and berberine against multidrug-resistant Klebsiella pneumoniae. J. Antibiot. 69 (10), 741-746. doi: 10.1038/ja.2016.15

Zhu, L., Zhang, D., Zhu, H., Zhu, J., Weng, S., Dong, L., et al. (2018). Berberine treatment increases Akkermansia in the gut and improves high-fat dietinduced atherosclerosis in Apoe-/- mice. Atherosclerosis 268, 117-126. doi: 10.1016/j.atherosclerosis.2017.11.023

Conflict of Interest: The authors declare that the research was conducted in the absence of any commercial or financial relationships that could be construed as a potential conflict of interest.

Copyright $\odot 2021$ Zhang, Wu, Yang, Chen, Liao, Zhu, Wu, Sun and Wang. This is an open-access article distributed under the terms of the Creative Commons Attribution License (CC BY). The use, distribution or reproduction in other forums is permitted, provided the original author(s) and the copyright owner(s) are credited and that the original publication in this journal is cited, in accordance with accepted academic practice. No use, distribution or reproduction is permitted which does not comply with these terms. 